\title{
Comparison on fine needle aspiration cytology (FNAC) and thyroid ultrasonography in management of thyroid nodules
}

\begin{abstract}
Introduction: Pathologies of the thyroid nodules are a common clinical problem in Sri Lanka. Clinical assessment, biochemical investigations, ultrasonography, Fine needle aspiration cytology (FNAC) and histology are used in their diagnosis. However, histology continues to remain as the gold standard. Accurate diagnoses of thyroid nodules are important in planning management and could result in major catastrophes if diagnosis is incorrect.
\end{abstract}

Objectives: To determine the ability of FNAC and ultrasonography (USS) independently and in combination to distinguish neoplastic nodules of thyroid from non-neoplastic nodules.

Methodology: A retrospective cross sectional study involving 73 patients from base hospital Embilipitiya, from 2009 January to 2013December was carried out.

Results: Study population was female predominant $70(95.9 \%)$ with their age ranging from 17-70 (mean 42.7). Sensitivity, specificity, positive predictive value, negative predictive value and accuracy for FNAC were $94.1 \%, 87.2 \%, 86.5 \%, 94.4 \%$, and $90.4 \%$ respectively. The corresponding values for ultrasonography were $64.7 \%$, $69.2 \%, 64.7 \%, 69.2 \%$ and $67.1 \%$ respectively.

Conclusion: The findings of this study are in keeping with the other studies of this country and the region. In addition FNAC has a higher validity than USS in detection of thyroid neoplasms.

Keywords: thyroid neoplasm, FNAC, thyroid ultrasound scan
Volume 5 Issue 2 - 2017

\author{
Gunaratne SA,' Wijesinghe HKSN,' \\ Wijesinghe CJ, 'Disanayake SKE,' \\ Godakandage MHP ${ }^{3}$ \\ 'Base Hospital Embilipitiya, Sri Lanka \\ ${ }^{2}$ University of Ruhuna, Sri Lanka \\ ${ }^{3}$ National Hospital of Colombo, Sri Lanka
}

Correspondence: Sandini A Gunaratne, Base Hospital,

Avissawella, Sri Lanka, Tel 07I2774538,

Email sandigunaratne@gmail.com

Received: June 24, 2017 | Published: October 05, 2017
Abbreviations: FNAC, fine needle aspiration cytology; CAT, chronic autoimmune thyroiditis text; TN, true negative; TP, true positive; FP, false positive; FN, false negative; SPSS, statistical package for the social sciences

\section{Introduction}

A nodular thyroid gland is a common occurrence, especially among females; although chances of these lesions being malignant are rare. ${ }^{1}$ In Sri Lanka, thyroid cancer is one of the five leading sites of cancer in females, and has a life time risk of $0.357 \%$ for both males and females. ${ }^{2}$ The gold standard for diagnosis of thyroid pathologies is histopathology. However it is important to correctly diagnose during initial investigations for management plans. The available investigations for diagnosis range from biochemical, cytological and imaging to histopathology. Of these, FNAC is one of the most accurate and cost-effective methods of evaluating thyroid nodules, which is further improved by incorporation of genetic and immunohistochemical tests. ${ }^{3,4}$ In recent studies sensitivity, specificity and accuracy of FNAC have been reported as high as 65-98\%, 72100, 95\% respectively. ${ }^{3}$ However The Royal College of Pathologists recommend a wide range of values for sensitivity, specificity and accuracy of FNAC with regard to detection of thyroid neoplasms. ${ }^{5}$ Thyroid FNAC has some limitations in cases of suspicious, inadequate, and indeterminate cytology and it is reported that, even in adequate cellular specimens, an "undetermined" result can occur in $4-15 \%$ of all cases. ${ }^{6}$ Therefore, a definitive distinction between neoplasms and non- neoplasms is difficult. In addition, there are falsepositives and false-negatives. Therefore, another investigation is used in combination to compensate for these limitations. Ultrasonography (USS) of the thyroid gland is considered as the most sensitive method for diagnosis of intrathyroidlesions in some research. ${ }^{7}$ The reported features of malignancy in USS include marked hypoechogenecity, presence of microcalcifications, irregular margins, nodule with shape taller than wide and intra-nodular hypervascularity in color Doppler. ${ }^{8}$ The presence of more than one of the above features, and combination of some features, increase the probability that a thyroid nodule represents a malignancy. ${ }^{8}$ In addition, it is non invasive, relatively inexpensive and widely available in Sri Lanka. Therefore it is necessary to assess the ability of FNAC and USS in diagnosis of thyroid pathologies in the local setting.

\section{Objectives}

To determine the ability of FNAC and USS independently and in combination to distinguish neoplastic nodules of thyroid from nonneoplastic nodules.

\section{Material and methods}

This is a retrospective cross sectional study involving patients with thyroid nodules who received treatment from Base Hospital Embilipitiya, from 2009 January to 2013 December. USS was done 
using a Toshiba Nemio XG and GP3machines with a linear array probe $10-15 \mathrm{MHz}$. FNACs were done using $23 \mathrm{G}$ needles and $10 \mathrm{ml}$ syringes and the smears fixed in 95\% alcohol. Haematoxaline and eosin was used for the staining of smears.

The sample was selected by convenient sampling method. Both multi nodular and solitary nodules were included. In case of multinodular goiters the largest or the ultrasonically recommended nodule was sampled. Surgery was done on that particular nodule or side if a hemithyroidectomy was performed. All nodules were not aspirated. The data was extracted from the FNAC, USS and histopathology reports of the patients who have undergone surgery during the study period.

\section{Data analysis}

All data was analyzed using statistical package for the social sciences (SPSS) (version 20.0) using descriptive statistics. Histological diagnosis was taken as the gold standard and the FNAC and USS diagnoses were compared with it.

\section{Definition of terms}

Cytological diagnosis was categorized into 4groups. A nonneoplastic FNAC includes colloid nodules, chronic autoimmune thyroiditis (CAT) andadenomatoid nodules. An indeterminate (follicular proliferations) FNAC includes a hyperplastic nodules and follicular neoplasms (follicular adenoma or follicular carcinoma) which could be either neoplastic or non-neoplastic. Neoplastic FNACs belong to the suspicious for malignancy or malignant categories. Suspicious for malignancy includes the FNACs in which criteria for malignancy are not fulfilled; when the varying cytological abnormalities associated with papillary carcinoma such as nuclear membrane irregularity, nucleolar abnormality, abnormal nucleus-tocytoplasm ratio were present, but did not fulfill all the criteria for a diagnosis of papillary carcinoma."Malignant" category included papillary carcinoma including variants (e.g.: follicular variant), follicular carcinoma, medullary carcinoma and anaplastic carcinoma or any other malignancy.

\section{Histological classification was as follows}

Neoplastic- follicular adenoma, follicular carcinoma, papillary carcinoma and variants and Hurthle cell adenoma Non neoplasticcolloid nodule, chronic autoimmune thyroiditis, hyper plastic nodule and adenomatoid nodule .Of these, benign histology includes colloid nodules, chronic autoimmune thyroiditis, adenomatoid nodules, Follicular tumor of unknown malignant potential and follicular adenomas and Hurthle cell adenomas. Malignant histology includes papillary carcinoma and variants, Follicular carcinoma and medullary carcinoma. Follicular tumours of unknown malignant potential (FTUMP) were considered as neoplastic since they have a $5-10 \%$ malignant risk. ${ }^{9}$ A true positive (TP) indicates a neoplastic lesion according to cytology and ultrasonography, which is confirmed by histology. A true negative (TN) indicates a non- neoplastic lesion according to cytology and histology which is confirmed by histology. A false positive (FP) has a neoplastic cytology/ultrasonography with non-neoplastic histology. A false negative (FN) has a neoplastic histology with anon neoplastic cytology/ultrasonography. Few of specimens contained papillary micro carcinoma (a focus less than $10 \mathrm{~mm}$ in greatest diameter). Micro carcinomas were categorized as a non neoplastic histology if there was no macroscopic cancer present, because the sub-centimeter foci would not be targeted by FNAC. In considering the inderminate cases of FNA diagnosis the comment which accompanied the report was taken into consideration and if the first differential diagnosis was ultimately the true histology it was taken as a TP.

\section{Results}

In the sample of 73 , mean age was 42.7 (range17-70) and majority $(22[30.1 \%])$ were in the $31-40$ year age group. The majority (70[95.9\%]) were females with a ratio of 1:0.04 Histologically non neoplastic conditions included colloid nodules(17(23.3\%), chronic autoimmune thyroiditis $(5,6.8 \%)$, hyper plastic nodule(15,20.5\%), FTUMP(1,1.4\%), contradictory we have said it was considered as malignant. The benign neoplasms were, follicular adenoma $(10,13.7 \%)$, Hurthle cell adenoma $(1,1.4 \%)$. The majority of the malignancies were papillary $(12[16.4 \%])$, followed by follicular variant of papillary $(5[6.8 \%])$ and follicular $(4[5.5 \%])$ and papillary micro carcinoma $(3[4.1 \%])$. Majority of the FNACs were follicular proliferations $(31,42.5 \%)$.Seventeen $(23.2 \%)$ were suspicious for a neoplasm. Non neoplastic FNACs include colloid nodule $(22,30.1 \%)$ and CAT $(3,4.1 \%)$. All 3 investigations agreed on the diagnosis in $63 \%(46)$ of cases. There were 3 cases of histological micro papillary carcinoma which were not considered in the accuracy calculation since they cannot be identified on FNAC or USS (Table 1-3).

Table I Comparison of FNAC and USS findings with the histological diagnosis

\begin{tabular}{|c|c|c|c|c|}
\hline & & Histology & & \\
\hline & & $\begin{array}{l}\text { Positive neoplastic } \\
\text { no }(\%)\end{array}$ & $\begin{array}{l}\text { Negative non-NEOPLASTIC } \\
\text { No }(\%)\end{array}$ & Total no (\%) \\
\hline & & $32(43.9)$ & $5(6.8)$ & $37(50.7)$ \\
\hline & Positive Neoplastic & $\mathrm{TP}$ & $\mathrm{FP}$ & \\
\hline FNAC & & $2(2.7)$ & $34(46.6)$ & $36(49.3)$ \\
\hline & Negative Non-neoplastic & $\mathrm{FN}$ & $\mathrm{TN}$ & \\
\hline & Total & 34 & 39 & $73(100.0)$ \\
\hline & Positive & $22(30.1)$ & $12(16.4)$ & $34(46.5)$ \\
\hline & Neoplastic & $\mathrm{TP}$ & FP & \\
\hline USS & Negative & $12(16.5)$ & $27(37)$ & $39(53.5)$ \\
\hline & Non-neoplastic & $\mathrm{FN}$ & $\mathrm{TN}$ & \\
\hline & Total & 34 & 39 & $73(100.0)$ \\
\hline
\end{tabular}

TP, true positive; $\mathrm{TN}$, true negative; $\mathrm{FP}$, false positive; $\mathrm{FN}$, false negative 
Table 2 Histological diagnosis of false positive and false negative

\begin{tabular}{|c|c|c|}
\hline & False positives & False negatives \\
\hline \multirow{4}{*}{ FNAC } & Colloid Nodule with Chronic Autoimmune Thyroiditis ${ }^{1}$ & \multirow{4}{*}{ Follicular Adenoma ${ }^{2}$} \\
\hline & Colloid Nodules ${ }^{2}$ & \\
\hline & Hyperplastic Nodules $^{1}$ & \\
\hline & colloid nodule, CAT and hyperplastic nodule ${ }^{1}$ & \\
\hline \multirow{5}{*}{ USS } & Hyperplastic Nodules ${ }^{4}$ & Follicular Adenomas ${ }^{5}$ \\
\hline & Colloid Nodules 5 & Follicular Carcinomas ${ }^{2}$ \\
\hline & $\mathrm{CAT}^{1}$ & Papillary Carcinoma ${ }^{1}$ \\
\hline & \multirow[t]{2}{*}{ CAT with Hyperplastic Nodule ${ }^{2}$} & Follicular variant of Papillary Carcinoma ${ }^{3}$ \\
\hline & & Hurthle cell Adenoma ${ }^{1}$ \\
\hline
\end{tabular}

CATL, chronic autoimmune thyroiditis

Table 3 Validity of FNAC and USS in diagnosing neoplastic lesions of the thyroid gland

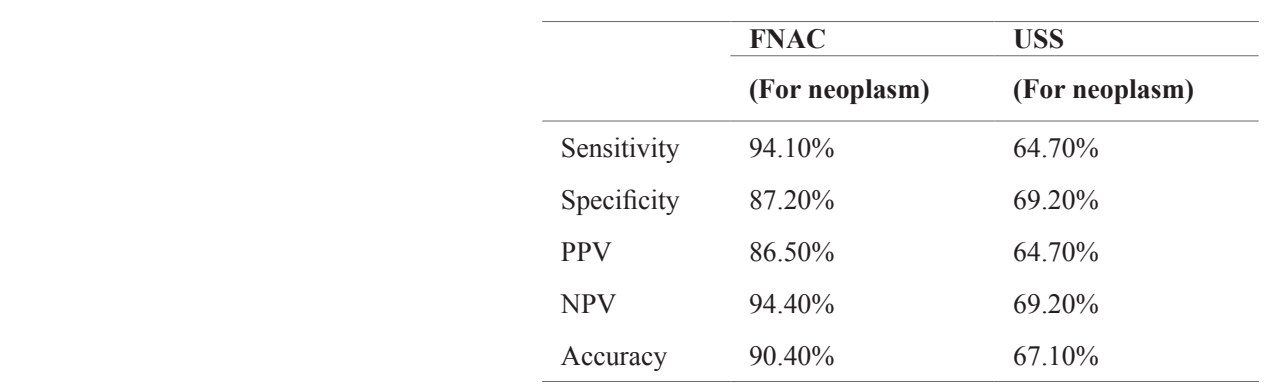

PPV, positive predictive value; NPV, negative predictive value

\section{Discussion}

Our study reveals that of the patients with thyroid nodule, a significant majority are females. This is further substantiated by many authors in their studies. ${ }^{9,10}$ However the increased concern on cosmesis and the higher health seeking behaviour in females may be additional factors favoring this. The age group with the highest number of subjects with thyroid disorders show a wide variation in different studies. ${ }^{11,12}$ Therefore our findings (31-40 years age group) are within the findings of the international studies (Table 4). There is a wide variation in the global statistics relating to validity of FNAC as a tool for diagnosing thyroid pathology and the Royal College of pathologists (RCPA- Australasia) has allowed a wide range for sensitivity, specificity of FNAC with regard to thyroid neoplasms. ${ }^{13,14}$ The sensitivity, specificity and accuracy for diagnosis of neoplasm by FNAC in our study are acceptable. However our study has a higher sensitivity than the regional studies. This may be due to the fact that the surgeries were mainly performed on FNAC results which were follicular proliferations, suspicious for malignancy or malignancies. Thus making the population biased and increasing the true positives. (Table 5) Our USS sensitivity is lower than the others. However Ozel et al. ${ }^{16}$ has excluded non diagnostic FNACs and patients with non diagnostic histology, while Yunus et al. ${ }^{15}$ has included only solid nodules and excluded the nodules with cystic components and toxic nodules. ${ }^{15,16}$ Thus their higher sensitivity can be explained.

Table 4 Comparison of our FNAC statistics with the other studies

\begin{tabular}{llllll}
\hline Study & Our study & Stephenson et al. ${ }^{{ }^{2}}$ & Beneragama et al. $^{13}$ & Priyani et al. $^{\mathbf{1 4}}$ & Basharat et al. $^{\mathbf{1 2}}$ \\
\hline Sensitivity & $94.10 \%$ & $65-98$ & 84.05 & 77.78 & 80 \\
Specificity & $87.20 \%$ & $76-100$ & 86.74 & 83 & 97.7 \\
PPV & $86.50 \%$ & - & 84.05 & 93.33 & 80 \\
NPV & $94.40 \%$ & - & 86.74 & 88.41 & 97.7 \\
Accuracy & $90.40 \%$ & $69-97$ & - & 90 & 96
\end{tabular}

PPV, positive predictive value; NPV, negative predictive value 
Table 5 Comparison of our USS statistics with the other studies

\begin{tabular}{|c|c|c|c|c|c|}
\hline \multirow[b]{2}{*}{ Study } & \multirow[b]{2}{*}{ Our study } & \multirow[b]{2}{*}{ Yunus et al. ${ }^{15}$} & \multicolumn{2}{|l|}{ Ozel et al. ${ }^{16}$} & \multirow[b]{2}{*}{ Kim et al. ${ }^{17}$} \\
\hline & & & Nodule $>1 \mathrm{~cm}$ & Nodule $=$ or $<1 \mathrm{~cm}$ & \\
\hline Sensitivity & $64.70 \%$ & $93.80 \%$ & $62.50 \%$ & $83.30 \%$ & $93.80 \%$ \\
\hline Specificity & $69.20 \%$ & $66 \%$ & $91.50 \%$ & $94.90 \%$ & $66 \%$ \\
\hline PPV & $64.70 \%$ & $56.10 \%$ & $30.30 \%$ & $62.50 \%$ & $56.10 \%$ \\
\hline NPV & $69.20 \%$ & $95.90 \%$ & $97.70 \%$ & $98.20 \%$ & $95.90 \%$ \\
\hline Accuracy & $67.10 \%$ & $74.80 \%$ & $89.90 \%$ & 93.8 & $74.80 \%$ \\
\hline
\end{tabular}

PPV, positive predictive value; NPV, negative predictive value

Even though less in numbers, false positives and negatives are a major concern. Regarding false positive and false negative FNACs the commonest reason is inability to distinguish follicular proliferations in CAT from neoplastic micro follicular proliferations. In addition some features of papillary carcinoma can be seen in CAT even in the absence of a neoplasm. ${ }^{17}$ To overcome this, RET /PTC mutation detection can be done to detect the papillary carcinomas. ${ }^{17}$ Mentioned below are some other reasons which account for FPs and FNs in thyroid FNAC. Hyper cellular specimens from follicular or Hurthle cell lesions may have features suggestive of, but not diagnostic for malignancy. Thus, these are diagnosed "suspicious for malignancy" and histology is necessary for definitive diagnosis. Small nodules less than $10 \mathrm{~mm}$ and larger nodules more than $40 \mathrm{~mm}$ may not have been accurately sampled in unguided FNACs.

Inadequate slide preparations, technical issues, interpretive errors can cause both false positives and negatives. In Sri Lanka general pathologists interpret both histology and cytology unlike in overseas where specialized cytopathologists diagnose FNAC. The False positives and negatives in USS findings could be due to a considerable overlap of characteristics in benign and malignant lesions in ultrasound. ${ }^{18}$ For example hypoechogenecity is suggestive of malignancy but benign nodules also can be hypo echoic. Neoplastic malignant nodules have irregular margins but in many studies 55\% of solid nodules have been shown to have irregular margins. ${ }^{18,20}$ In a multinodular thyroid, mistaking diffusely infiltrative thyroid carcinomas and multifocal carcinomas for benign disease, and failing to recognize microcalcifications in papillary thyroid cancer can result in errors. Potential diagnostic pitfalls include routinely dismissing small nodules, assuming that multiple nodules are most likely benign, mistaking carcinomas for cystic hyper plastic nodules and Graves' disease, misdiagnosing cystic or calcified nodal metastases for nodules and misinterpreting adjacent nodal metastasis for benign thyroid nodules. ${ }^{19}$

\section{Conclusion and recommendations}

The findings reveal that the statistics of our study are in keeping with the other studies of this country and the region. In addition FNAC has a higher sensitivity and specificity than the USS in detection of thyroid neoplasms.

\section{Limitations}

It is not $100 \%$ certain that the nodule that was scanned was biopsied in benign cases, however in the case of suspicious/malignant nodules it was identified. However in both cases that particular nodule was surgically excised.

Therefore we recommend the following for further improvement.

i. USS guided FNACs at all times- especially for all small $(<10 \mathrm{~mm})$ and single suspicious lesions in a multinodular goiter. ${ }^{21}$

ii. To improve sampling, aspirates should be obtained from multiple sites of the nodule rather than repeatedly from a single site; especially in a large nodule.

iii. Clinical assessment, FNAC diagnosis and USS diagnosis should always be considered in combination to improve the quality of the management plan for thyroid nodules.

\section{Acknowledgements}

None.

\section{Conflict of interest}

The author declares no conflict of interest.

\section{References}

1. Razmpa, Ghanaati H, Naghibzadeh B, et al. Comparison of ultrasound findings with cytologic results in Thyroid nodules. Acta Medica Iranica. 2002;40(3):146-151

2. National Cancer Control Programme. Cancer Incidence data: Sri Lanka. 9th ed. Ministry of health Sri Lanka; 2007.

3. Dean DS, Gharib H. Fine-needle aspiration biopsy of the thyroid gland. Endo text [Internet]. 2000.

4. Ogilvie JB, Piatigorsky EJ, Clark OH. Current status of fine needle aspiration for thyroid nodules. Adv Surg. 2006;40:223-238.

5. Stephenson TJ, Johnson SJ. Guidance on the reporting of thyroid cytology specimens; 2016

6. Bonzanini M, Amadori P, Morelli L, et al. Subclassification of the grey zone of thyroid cytology; a retrospective descriptive study with clinical, cytological, and histological correlation. J Thyroid Res. 2011;2011:251680. 
7. Solbiati L, Charboneau JW, Osti V, et al. The thyroid gland. In: Rumack CM, et al. editors. Diagnostic Ultrasound. 3rd ed. St Louis, Missouri USA; 2010. p. 735-770.

8. Cooper DS, Doherty GM, Haugen BR, et al. Revised American Thyroid Association management guidelines for patients with thyroid nodules and differentiated thyroid cancer. Thyroid. 2009;19(11):1167-1214.

9. Elaraj DM. Evaluation of the thyroid nodule. USA: Springer science \& business media; 2009. p. 23-35.

10. Menegassi J, Bordin V, Oliveira SS. Diagnostic accuracy between fine needle aspiration biopsy (FNA) and pathological examination of the thyroid. Brazilian Journal of Pathology and Laboratory Medicine. 2013;49(4):288-293.

11. Chandanwale S, Singh N, Kumar H, et al. Clinicopathological correlation of thyroid nodules. International Journal of Pharmaceutical and Biomedical Research. 2012;3(3):97-102.

12. Basharat R, Bukhari MH, Saeed S, et al. Comparison of fine needle aspiration cytology and thyroid scan in solitary thyroid nodule. Patholog Res Int. 2011;2011:754041.

13. Beneragama DH, Jayasuriya W, Samarawickrama RIU, et al. Validity of fine needle aspiration cytology in the diagnosis of thyroid diseases. Journal of Diagnostic Pathology. 2006;5:19-29.

14. Priyani AAH, Ileperuma A, Fernando R. Validity of fine needle aspiration cytology in diagnosing thyroid gland neoplasms. Journal of Diagnostic Pathology. 2011;1(6):34-41.
15. Yunus M, Ahmed Z. Significance of ultrasound features in predicting malignant solid thyroid nodules: Need for fine-needle aspiration. The Journal of the Pakistan Medical Association. 2010;60(10):848-853.

16. Ozel A, Erturk SM, Ercan A, et al. The diagnostic efficiency of ultrasound in characterization for thyroid nodules: how many criteria are required to predict malignancy? Med Ultrason. 2012;14(1):24-28.

17. De Silva MVC. Diagnosis of thyroid disease- the role of the pathologist. Ceylon Medical Journal. 2007;52(1):4-6.

18. Kim EK, Park CS, Chung WY, et al. New sonographic criteria for recommending fine-needle aspiration biopsy of nonpalpable solid nodules of the thyroid. AJR Am J Roentgenol. 2002;178(3):687-691.

19. Avinash B, Ahmad N, Sreedevi T. Role of ultrasonography to differentiate benign and malignant thyroid nodules in correlation with fine needle aspiration. International J of Scientific study. 2015;4(5):81-87.

20. Yi K. The revised 2016 Korean Thyroid association Guidelines for Thyroid nodules and cancers: differences from 2015American thyroid association guidelines. Endocrinol Metab (Seoul). 2016;319(3):373-378.

21. Tamas Solymosi, Gyula Lukacs Toth, Laszlo Budai, et al. The clinical and pathological presentation of thyroid nodules in children and the comparison with adult population: experience of a single institution. Int J Endocrinol. 2016;2016:1256189. 